\title{
On Kalman Filtering With Nonlinear Equality Constraints
}

\author{
Simon J. Julier, Member, IEEE, and Joseph J. LaViola, Jr., Member, IEEE
}

\begin{abstract}
The state space description of some physical systems possess nonlinear equality constraints between some state variables. In this paper, we consider the problem of applying a Kalman filter-type estimator in the presence of such constraints. We categorize previous approaches into pseudo-observation and projection methods and identify two types of constraints-those that act on the entire distribution and those that act on the mean of the distribution. We argue that the pseudo-observation approach enforces neither type of constraint and that the projection method enforces the first type of constraint only. We propose a new method that utilizes the projection method twice-once to constrain the entire distribution and once to constrain the statistics of the distribution. We illustrate these algorithms in a tracking system that uses unit quaternions to encode orientation.
\end{abstract}

Index Terms - Kalman filtering, quaternions, measurement matrix, nonlinear constraints.

\section{INTRODUCTION}

$\mathbf{S}^{\mathrm{o}}$ OME physical systems have equality constraints between their state variables. These constraints can arise for several reasons. Some constraints arise from the basic laws of physics. The mass of constituents in a sealed chemical reactor, for example, must remain constant throughout the reaction process [1]. Other constraints arise from the mathematical description of a state vector. If the state is modelled as a rotation matrix, the rows of the matrix must be orthonormal [2]. Constraints can arise from kinematic or geometric considerations of a system. The coordinated turn model for an aircraft, for example, assumes that the acceleration vector is orthogonal to the velocity vector [3], [4].

A number of approaches have been developed to apply these equality constraints within the Kalman filter (KF) framework. These can be broadly classified into pseudo-observation, projection and reparameterization methods. The pseudo-observation method creates a fictitious observation whose variance is zero [3]-[9]. By substitution, it can be shown that the KF update equations project the state estimate onto the constraint sur-

\footnotetext{
Manuscript received October 26, 2005; revised September 18, 2006. This work was supported in part by the Office of Naval Research, the Department of Energy, and Microsoft. The associate editor coordinating the review of this manuscript and approving it for publication was Dr. Petr Tichvsky.

S. J. Julier was with the 3D Mixed and Virtual Environments Laboratory, Naval Research Laboratory, Washington, DC 20375 USA. He is now with the Computer Science Department, University College London, London, U.K. (e-mail: s.julier@cs.ucl.ac.uk.).

J. J. LaViola, Jr. is with the School of Electrical Engineering and Computer Science, University of Central Florida, Orlando, FL 32816 USA (e-mail: jj1@ cs. ucf.edu).

Color versions of one or more of the figures in this paper are available online at http://ieeexplore.iee.org.

Digital Object Identifier 10.1109/TSP.2007.893949
}

face [7]. The projection approach constructs a projection operator that transforms the estimate so that it lies on the constraint surface [5], [10]-[12]. The reparameterization approach simply reparameterizes the system so that the equality constraint is not required [13]. Since the focus of this work is on examining the effects of different algorithms for constrained estimation, the reparameterization approach does not fall within the scope of this paper. ${ }^{1}$ Thus, we focus on the first two methods.

In this paper, we argue that although the pseudo-observation and projection approaches have clear, simple, intuitive interpretations when the constraints are linear, none of these properties hold when the constraints are nonlinear. The main reason is that both approaches implicitly assume that if the probability distribution obeys the nonlinear constraint, then the mean of the distribution (which is the property maintained in the filter) obeys the constraint as well. The pseudo-observation method makes the further assumption that the Kalman filter update rule, which is a linear projection operator, is sufficient to constrain a probability distribution to a nonlinear surface.

To overcome these difficulties, we propose a two-step constraint application method. The first step applies the projection method to the unconstrained estimate. As a result, the probability distribution of the estimate is constrained to lie along the constraint surface. This causes the covariance in the estimate to decrease. In the second step, the distribution is translated so that its mean lies on the constraint surface. This, in turn, causes the covariance in the estimate to increase.

The structure of this paper is as follows. The problem statement is described in Section II. Section III introduces an illustrative example which is used throughout much of this paper: estimating an angle of rotation about a single axis using complex numbers. We analyze the pseudo-observation and projection methods and show that they fail. Section IV examines the properties of the constraints in detail and identifies two types of constraints. The first type is a constraint on each sample in the entire distribution. The second type is a constraint on just the mean of the estimate. We show that neither the pseudo-observation nor the projection approaches fully satisfies either constraint interpretation. Section V derives the new two-step approach for applying constraints. In Section VI, the new algorithm is illustrated in a head tracking application that uses unit quaternions to represent orientation. Conclusions are drawn in Section VII.

\footnotetext{
${ }^{1}$ We assume that if a system has a nonlinear constraint, the designer has determined that this is an appropriate representation to use. In addition, the comparison between reparameterization and using the constraint algorithms is likely to be highly system dependent.
} 


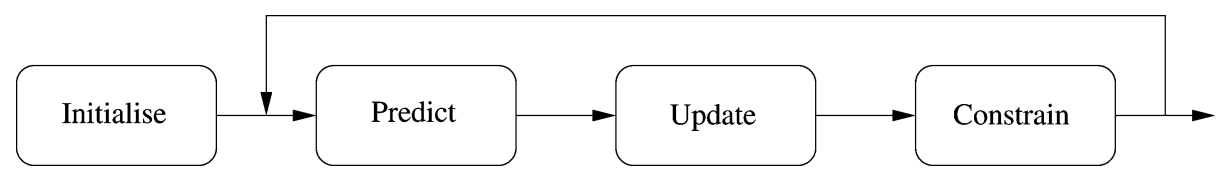

Fig. 1. Constrained filter: The prior estimate is predicted to give $\hat{\mathbf{x}}(k \mid(k-1))$. The filter is updated with the observation to give the unconstrained estimate $\hat{\mathbf{x}}^{*}(k \mid k)$. The constraint is applied to give the final estimate $\hat{\mathbf{x}}(k \mid k)$.

\section{Problem Statement}

We seek the minimum-mean squared error estimate of the state vector of the nonlinear discrete time system

$$
\begin{aligned}
& \mathbf{x}(k)=\mathbf{f}[\mathbf{x}(k-1), \mathbf{u}(k), \mathbf{v}(k)] \\
& \mathbf{z}(k)=\mathbf{h}[\mathbf{x}(k), \mathbf{u}(k), \mathbf{w}(k)]
\end{aligned}
$$

where $\mathbf{x}(k-1)$ is the state of the system at time step $k-1, \mathbf{u}(k)$ is the control input vector, $\mathbf{v}(k)$ is the noise process due to disturbances and modelling errors, $\mathbf{z}(k)$ is the observation vector, and $\mathbf{w}(k)$ is measurement noise. It is assumed that the noise vectors $\mathbf{v}(k)$ and $\mathbf{w}(k)$ obey the usual zero mean and independence assumptions.

An equality constraint exists between the state variables which can be written in the form

$$
\mathbf{c}[\mathbf{x}(k)]=\mathbf{0}
$$

Furthermore, a projection function $\mathbf{p}[\mathbf{x}(k)]$ exists such that

$$
\mathbf{c}[\mathbf{p}[\mathbf{x}(k)]]=\mathbf{0}
$$

for all values of $\mathbf{x}(k)$.

We use the notation that the estimate at time step $i$, using all observations up to time step $j$, is the random vector $\mathbf{x}(i \mid j)$ with mean $\hat{\mathbf{x}}(i \mid j)$ and covariance $\hat{\mathbf{P}}(i \mid j)$. We require the condition that the estimate be consistent [14]. In other words

$$
\mathbf{P}(i \mid j)-\mathrm{E}\left[\tilde{\mathbf{x}}(i \mid j) \tilde{\mathbf{x}}^{T}(i \mid j)\right] \geq \mathbf{0}
$$

where $\tilde{\mathbf{x}}(i \mid j)=\mathbf{x}(i \mid j)-\hat{\mathbf{x}}(i \mid j)$ is the error in the estimate, and $\geq \mathbf{0}$ means that the result is positive semidefinite.

The constraint is to be applied using the architecture shown in Fig. 1: The filter is initialized, predicted, and updated with the measurement to give an unconstrained estimate $\hat{\mathbf{x}}^{*}(k \mid k)$ with covariance $\mathbf{P}^{*}(k \mid k)$. The constraint is applied, and the resulting estimate $\{\hat{\mathbf{x}}(k \mid k), \mathbf{P}(k \mid k)\}$ obeys the constraint

$$
\mathbf{c}[\hat{\mathbf{x}}(k \mid k)]=\mathbf{0} .
$$

This form is consistent with Alouani's suggestion: The constraint is only applied to the updated estimate and is thus likely to be most accurate[4]. ${ }^{2}$

\footnotetext{
${ }^{2}$ Exactly the same approach was subsequently proposed by Simon [8].
}

In the special case where the constraints are linear, the constraint and projection equations are [5]

$$
\begin{aligned}
\mathbf{c}[\mathbf{x}(k)]= & \mathbf{C x}(k)-\mathbf{c} \\
\mathbf{p}[\mathbf{x}(k)]= & \mathbf{x}(k)-\mathbf{D C}^{T}\left(\mathbf{C D C}^{T}\right)^{-1} \\
& \times(\mathbf{c}-\mathbf{C x}(k))
\end{aligned}
$$

where $\mathbf{C}$ and $\mathbf{c}$ define the linear constraint (such that $\mathbf{C x}=\mathbf{c}$ ), and $\mathbf{D}$ is a positive semidefinite weighting matrix that can be chosen to, for example, minimize $|\mathbf{P}(k \mid k)|$.

A number of approaches for estimation in the presence of these constraints have been derived and, as explained in the introduction, we consider the the pseudo-observation and the projection methods.

\section{A. Pseudo-Observation Method}

The pseudo-observation method generates a fictitious observation from the constraint function. The observation model is

$$
\mathbf{z}(k)=\mathbf{c}[\mathbf{x}(k)] .
$$

The value observed from the system is always treated as $\mathbf{0}$ with variance $\mathbf{0}$. Using the EKF the update is

$$
\begin{aligned}
\hat{\mathbf{x}}(k \mid k) & =\hat{\mathbf{x}}^{*}(k \mid k)-\mathbf{W}(k) \mathbf{c}\left[\hat{\mathbf{x}}^{*}(k \mid k)\right] \\
\mathbf{P}(k \mid k) & =\mathbf{P}^{*}(k \mid k)-\mathbf{W}(k) \mathbf{S}(k) \mathbf{W}^{T}(k)
\end{aligned}
$$

where

$$
\begin{aligned}
\mathbf{S}(k) & =\boldsymbol{\nabla} \mathbf{c} \mathbf{P}^{*}(k \mid k) \nabla^{T} \mathbf{c} \\
\mathbf{W}(k) & =\mathbf{P}^{*}(k \mid k) \nabla^{T} \mathbf{c S}^{-1}(k)
\end{aligned}
$$

and $\nabla \mathbf{c}$ is the Jacobian of $\mathbf{c}$ evaluated about $\hat{\mathbf{x}}^{*}(k \mid k) .^{3}$

This approach was first proposed Tahk [3], who considered the problem of applying linear equality constraints and then demonstrated a linearized version on a coordinated turn example. Chia derived the same result when he extended his batch constrained estimation scheme [15] to a recursive form to estimate the parameters of electrically stimulated muscles [5]. Doran proposed exactly the same method for economic systems and showed how it could be used to estimate the determinants of exchange rate and population growth rates in Australia [6]. Wen showed that the pseudo-measurement approach was equivalent to projecting the estimate to the subspace where the constraint was guaranteed to hold [7]. Takh, Doran, and Wen all argued that extending the method to nonlinear systems is straightforward and analogous to the EKF: Simply linearize

\footnotetext{
${ }^{3}$ This approach can only be used if $\mathbf{S}(k)$ is invertible. This is normally ensured by the injection of process noise of the appropriate structure in the prediction step.
} 
all the nonlinear equations. To offset linearization errors, they recommend modifying the constraint equation so that the covariance on the pseudo-observation is a non-zero value. Alouani considered the application of nonlinear constraints to study the effect of target kinematics [4]. To compensate for linearization errors and to account for the fact that he was applying a motion heuristic, he introduced an adaptive weighting scheme that allowed the noise to decay slowly through time. This causes the constraint to be applied progressively more tightly.

However, to the best of our knowledge, Geeter was the first (and only) author to explicitly consider the issue that nonlinear equality constraints are fundamentally different from linear equality constraints. He argued that nonlinear constraints are plagued by two sources of errors: truncation errors and base point errors [9]. Truncation errors arise because the statistics of the distribution, which has undergone a nonlinear transformation, cannot, in general, be calculated precisely. Rather, a lower order approximation (such as a Taylor Series expansion truncated to a particular order) must be used. Base point errors occur because the filter linearizes around the estimated value of the state rather than the true value. As a result, the constraint surface is not oriented correctly. To overcome these difficulties, Geeter developed an algorithm known as the Smoothly Constrained Kalman Filter (SCKF) [13]. This algorithm transforms hard constraints into soft constraints and provides an exponential weighting term that progressively tightens the constraints.

All of these methods require the injection of stabilizing process noise into the constraint process, meaning that the constraint is loose: The state is not guaranteed to obey the constraint value. In some cases, this is acceptable. In the applications developed by Takh and Alouani, for example, they were applying target motion heuristics. However, this approach is not appropriate when strict mathematical constraints must be applied. In this situation, the projection approach can be used.

\section{B. Projection Approach}

The projection approach applies the projection function directly to the state estimate. For example, using the EKF, the constrained mean and covariance are given by

$$
\begin{aligned}
\hat{\mathbf{x}}(k \mid k) & =\mathbf{p}\left[\hat{\mathbf{x}}^{*}(k \mid k)\right] \\
\mathbf{P}(k \mid k) & =\boldsymbol{\nabla} \mathbf{p} \mathbf{P}^{*}(k \mid(k-1)) \nabla^{T} \mathbf{p}
\end{aligned}
$$

where $\nabla \mathbf{p}$ is the Jacobian of $\mathbf{p}$ and is calculated about $\hat{\mathbf{x}}^{*}(k \mid k)$. From the definition of the projection function in (2), the constraint is guaranteed to hold true.

Despite its simplicity, relatively few authors have used this approach. Nihan considered the problem of estimating origin-destination matrices for road networks [11]. To conserve the number of vehicles, the rows of the matrix must sum to one. Nihan constrained the estimate by dividing each row by its sum. Azuma considered the problem of estimating the pose of a human head [10]. He used quaternions to estimate the attitude. To guarantee the normalization constraint, the quaternion states were divided by their length. In considering the problem of enforcing the orthonormal properties of rotation matrices, Choukroun proposed a method known as optimal brute force orthogonalization, which is used to find the closest (in the Frobenius norm sense) orthonormal matrix to a given matrix [16]. He showed that this was equivalent to the orthogonal Procrustes problem and that its closed form solution can be expressed in terms of a projection operation. Durrant-Whyte considered the problem of applying coordinate transformations to parameterized geometric objects [17]. Rigid body transformations introduce constraints between configurations of points (such as the distances between points do not change). Recently, Yang considered the problem of tracking ground vehicles [12]. Nonlinear constraints arise when the state estimates are constrained to lie on curved roads. He introduced a nonlinear projection method for second order constraints using Lagrange multipliers.

Although the pseudo-observation and projection methods share the property that they project the state estimate to the constraint surface, they are qualitatively different. The pseudo-observation method uses the Kalman filter's linear update rule. Therefore, the projection operator is linear and its parameters are chosen to minimize the mean squared error estimate. The projection method can utilize any projection operator that is consistent with (2). However, if this operator takes no account of the covariance matrix, it can actually cause the covariance to increase [5].

We now illustrate these approaches in a simple example.

\section{EstimATING ANGLES}

Consider the problem of estimating an angle of rotation about a single axis. One (non-minimal) way to represent this rotation is to use complex numbers

$$
\mathbf{x}=x+i y
$$

where $\|\mathbf{x}\|=1$.

Fig. 2 shows a set of unconstrained estimates whose mean and covariances do not satisfy the equality constraint. Each estimate was generated from calculating the mean and covariance of a Monte Carlo sample of $4 \times 10^{6}$ samples of

$$
\mathbf{x}=\cos \theta+i \sin \theta
$$

where $\theta$ is a normally distributed random vector for eight different values of the mean (steps of $\pi / 4$ ) and standard deviation $\sigma_{\theta}=\pi / 8$. These unconstrained estimates have several important properties.

1) Each individual sample satisfies the nonlinear constraint $\|\mathbf{x}\|=1$.

2) The mean of each distribution has the property $\|\hat{\mathbf{x}}\|<1$. This is a direct consequence of Jensen's Inequality [18], where the constraint is convex, and therefore, its mean value (for all non-zero values of the covariance) must lie within the constraint surface.

3) Although the distribution is oriented so that the major axis is orthogonal to the radial line that passes through the mean, the covariance is not singular. The reason is that although $x$ and $y$ are not independent, the relationship 


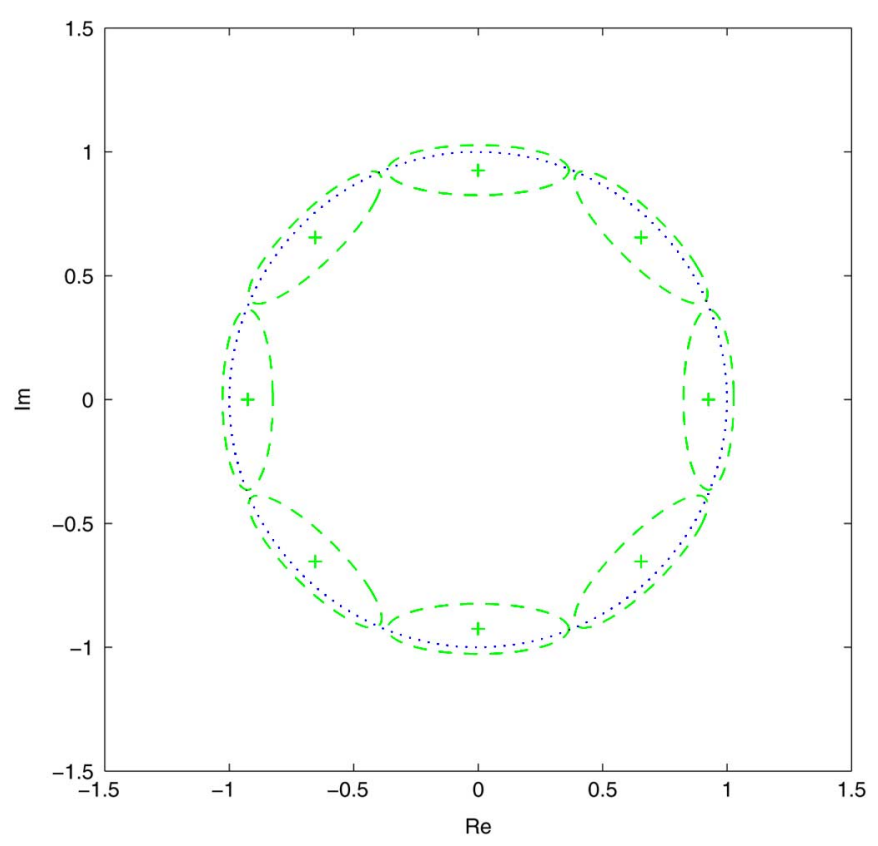

Fig. 2. Unconstrained estimates. The mean values lie at + , and the covariances ( $1 \sigma$ values) are represented by the dashed ellipse. The unit circle is shown using dotted lines.

is nonlinear and is not fully described by the correlation structure (which is a first order parameterization of dependency).

We now illustrate the effect of the constraint algorithms in this example.

\section{A. Pseudo-Observation}

The constraint function $\mathbf{c}[\mathbf{x}]$ can be written as

$$
\mathbf{c}[\mathbf{x}]=x^{2}+y^{2}-1=0
$$

Applying a single update does not lead to a normalized estimate after a single iteration. The reason is that the EKF can be considered to be a single step in an optimization algorithm [19]. Therefore, an iterated EKF [20] was applied with a threshold of $10^{-3}$ on the change in the normalized estimate between successive updates. Each update required no more than eight iterations.

The normalized estimates are shown in Fig. 3. At first sight, this result seems reasonable: The normalized estimates lie on the unit circle, showing that the estimate satisfies the constraint. Furthermore, the effect of the constraint has been reflected in the covariance matrix: It has collapsed so that all of the uncertainty now lies on the tangent to the unit circle. However, this result is not mathematically reasonable because the uncertainty is incompatible with the constraint surface. Consider the constrained estimate that lies at $(1,0)$. Because the variance is non-zero, there is some uncertainty in this estimate, and so, the true value can lie at a point where $y \neq 0$. Because the magnitude of the estimate is 1 , the magnitude of $x$ must decrease as the magnitude of $y$ increases. However, the covariance matrix

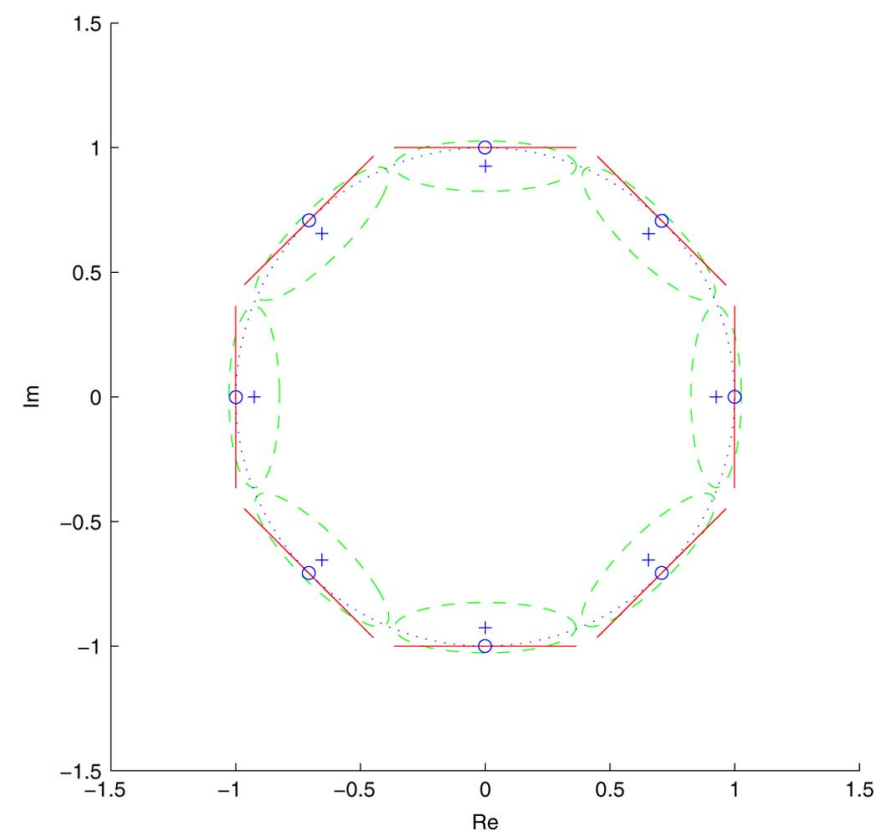

Fig. 3. Unconstrained and constrained estimates using the linearized pseudoobservation method. The unconstrained estimates are the set of dashed ellipses with means at + . The constrained estimates are shown as solid ellipses (collapsed to lines because the covariances are singular) with means at 0 . The unit circle is drawn using a dotted line.

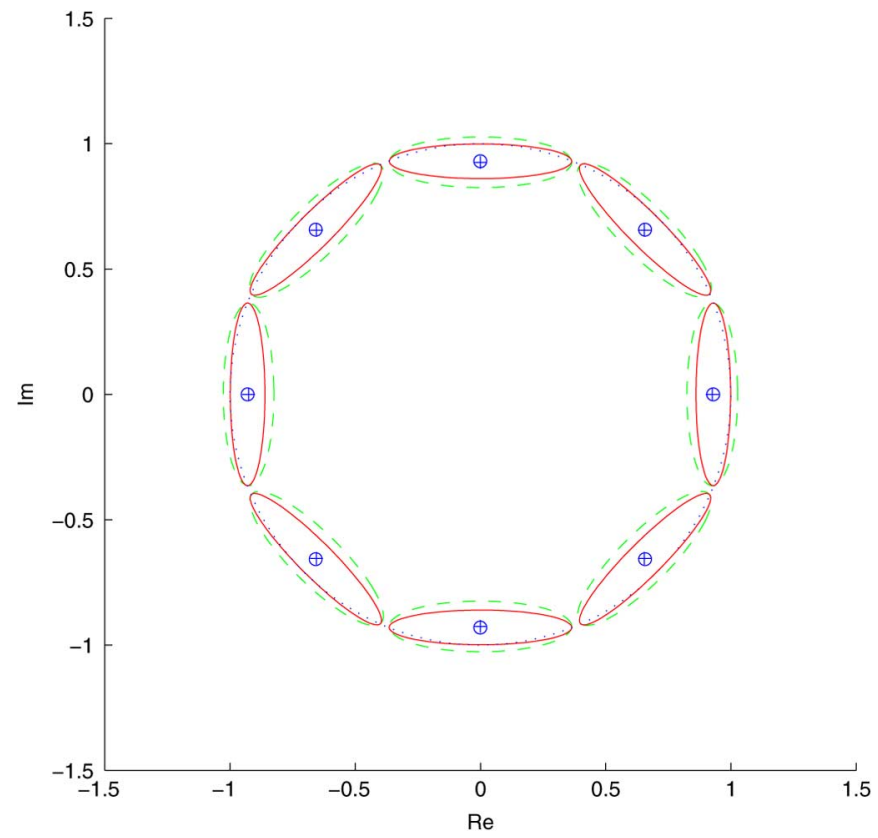

Fig. 4. Unconstrained and constrained estimates using the second order UT pseudo-observation algorithm. The constrained estimates are the set of dashed ellipses with means at + . The constrained estimates are shown as solid ellipses with means at $o$. The unit circle is drawn as the dotted line.

implies that the value of $x$ is known perfectly, and therefore, its value cannot change.

Geeter proposed that nonlinear constraints are different in two ways: truncation and base-point errors [9]. To test Geeter's first hypothesis, we utilized the second order Unscented Transformation (UT) [21]. The UT implicitly calculates the second and higher order terms in the Taylor series expansion about the prior mean and should thus lead to a more accurate estimate. Since 


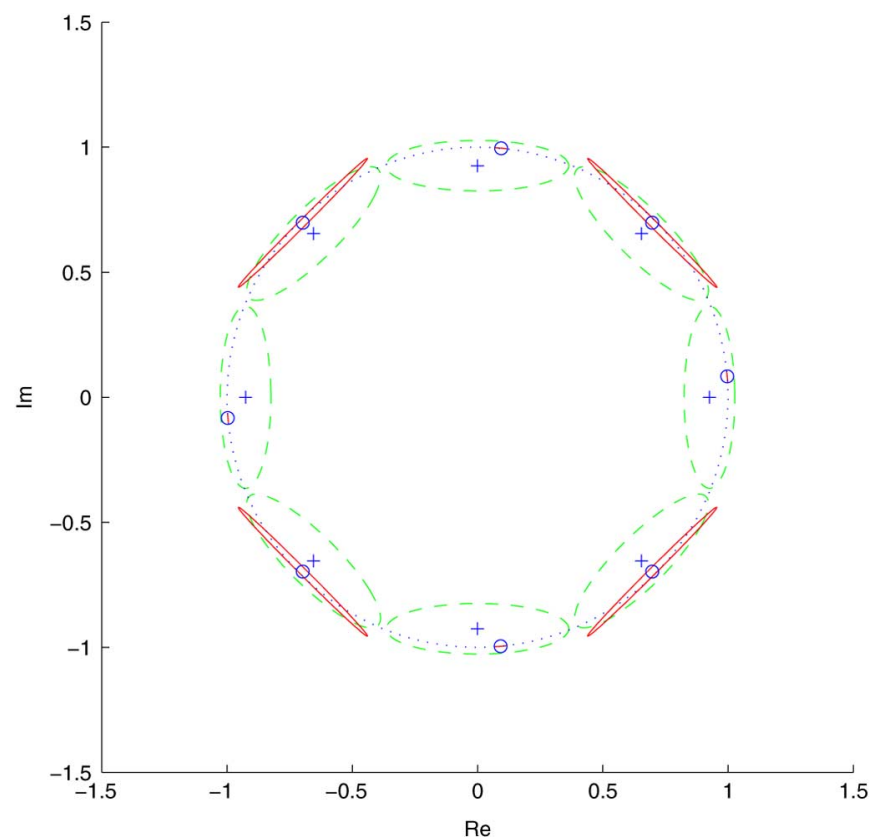

Fig. 5. Unconstrained and constrained estimates using the SCKF algorithm. The unconstrained estimates are the set of dashed ellipses with means at + . The constrained estimates are shown as solid ellipses (collapsed to short solid lines in some cases) with means at 0 . The unit circle is drawn with a dotted line.

this is a 2-D example, a value of $\kappa=1$ leads to the result that the mean is calculated precisely, and the covariance is calculated correctly to the second order. The update was repeatedly applied until the magnitude of the difference between successive estimates was $10^{-3}$. The results are shown in Fig. 4. Although the covariance matrices are not singular, the mean values have not changed significantly from their original unnormalized state.

To test Geeter's second hypothesis, we applied the SCKF. Fig. 5 shows the results using Geeter's recommended value $s_{k \text {,threshold }}^{c}=100$. Surprisingly, the algorithm fails to converge to a solution when the nominal angle aligns with one of the coordinate axes. ${ }^{4}$ For the results that lie off the coordinate axes, the results appear slightly better: The covariance matrices are non-singular. However, the constraint is not obeyed perfectly-the norm is 0.984 instead of 1.0.

\section{B. Projection}

The projection function we apply is ${ }^{5}$

$$
\mathbf{x}=\mathbf{x}^{*} /\left\|\mathbf{x}^{*}\right\|
$$

Fig. 6 plots the results using linearization. The constraint is automatically satisfied, and no iterative scheme is required. However, once again, the estimate covariance matrix is singular, and thus, this method suffers from the same difficulties as the pseudo-observation update. Fig. 7 shows the results when the UT is applied. The results are similar to those in Fig. 4: The

${ }^{4} \mathrm{We}$ believe this is because the SCKF, like the other pseudo-observation methods, actually requires stabilizing noise to be injected into state estimate. The correct implementation of the SCKF should interleave a single iteration of the constraint equation between the normal Kalman filter prediction-update cycles [22].

${ }^{5}$ This function, rather than $y=\sqrt{1-x^{2}}$, was chosen because both $x$ and $y$ are guaranteed to be real for all $\|\mathbf{x}\|>0$.

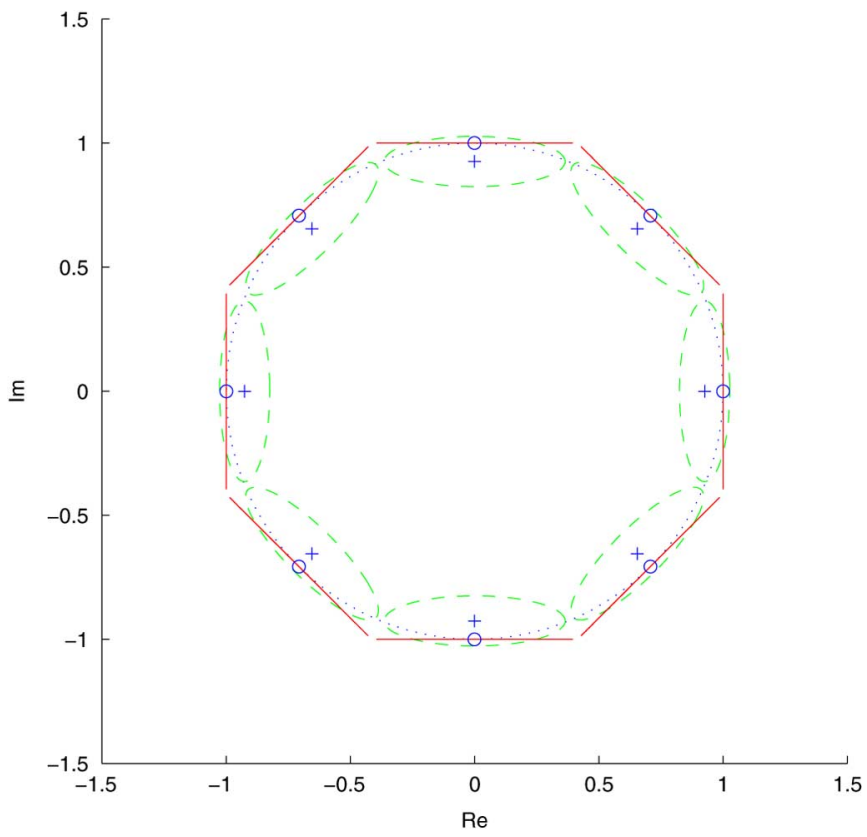

Fig. 6. Unconstrained and constrained estimates using the linearized projection algorithm. The unconstrained estimates are the set of dashed ellipses with means at + . The constrained estimates are shown as solid ellipses with means at 0 . The unit circle is drawn as the dotted line.

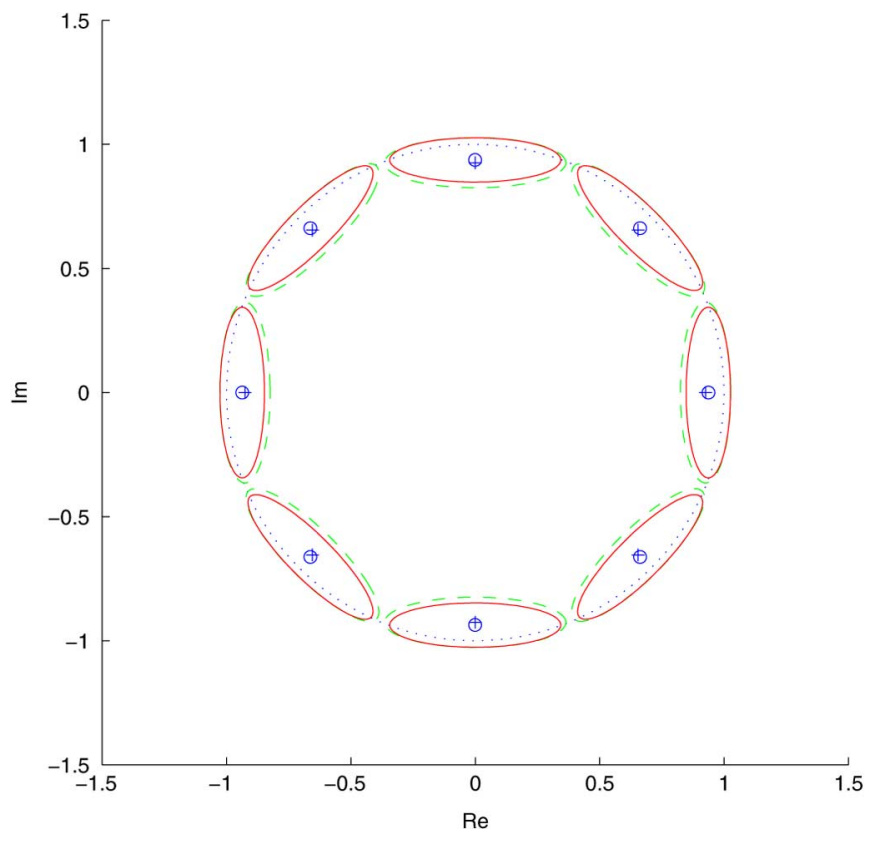

Fig. 7. Unconstrained and constrained estimates using the UT projection algorithm. The unconstrained estimates are the set of dashed ellipses with means at + . The constrained estimates are shown as solid ellipses with means at 0 . The unit circle is drawn with the dotted line.

constrained mean does not move significantly from the unconstrained mean. However, the covariance is now slightly larger.

This example illustrates that both the pseudo-observation and projection approaches lead to undesired behaviors: The linearized approaches lead to singular covariance matrices; the higher order versions of these transformations lead to mean estimates whose values do not change; Geeter's algorithm leads to an unnormalized estimate or an estimate with zero variance. We believe that this example is not an isolated special case but 
arises from a misunderstanding as to what constraint is being satisfied.

\section{Meaning OF CONSTRAints}

We believe that the difficulties illustrated in the previous section arise from the fact that there are, in fact, two types of constraints that can be defined. The first type-Type I constraints-apply a constraint to each point of the distribution

$$
\mathbf{c}[\mathbf{x}(k \mid k)]=\mathbf{0} .
$$

Type II constraints are constraints on the estimate propagated by the Kalman filter. These are normally posed as constraints on the mean estimate

$$
\mathbf{c}[\hat{\mathbf{x}}(k \mid k)]=\mathbf{0} .
$$

Type I constraints provide information about the entire shape of the probability distribution of $\mathbf{x}(k)$. This provides additional information and reduces the uncertainty in the distribution. A Type II constraint, however, only specifies information about the expected value of the distribution of $\mathbf{x}(k)$; no other information is provided. Therefore, a Type II constraint does not provide sufficient information to reduce the uncertainty in the distribution.

The relationship between the two types is laid out in the following theorem.

Theorem 1: The Type I constraint subsumes the Type II constraint only if the constraint is linear.

Proof: Assume the Type I constraint holds. Ignoring the time index for convenience, each sample in the constrained distribution $\mathbf{x}$ obeys the property

$$
\mathbf{c}[\mathrm{x}]=0 .
$$

Equivalently, each sample can be obtained from

$$
\mathrm{x}=\mathrm{p}\left[\mathrm{x}^{*}\right]
$$

where $\mathrm{x}^{*}$ is a sample in the unconstrained distribution. Now, the Type II constraint is obeyed if

$$
\mathbf{c}[\hat{\mathbf{x}}]=0
$$

However, since

$$
\hat{\mathbf{x}}=\mathrm{E}\left[\mathbf{p}\left[\mathbf{x}^{*}\right]\right]
$$

$\hat{\mathbf{x}}$ obeys the Type II constraint if it can be written as

$$
\hat{\mathbf{x}}=\mathbf{p}\left[\overline{\mathbf{x}}^{*}\right] .
$$

Therefore, the Type I constraint subsumes the Type II constraint if there exists a $\overline{\mathbf{x}}^{*}$ such that

$$
\mathbf{p}\left[\overline{\mathbf{x}}^{*}\right]=\mathrm{E}\left[\mathbf{p}\left[\mathbf{x}^{*}\right]\right] .
$$

In general, this can only be satisfied if $\mathbf{p}$ is linear.

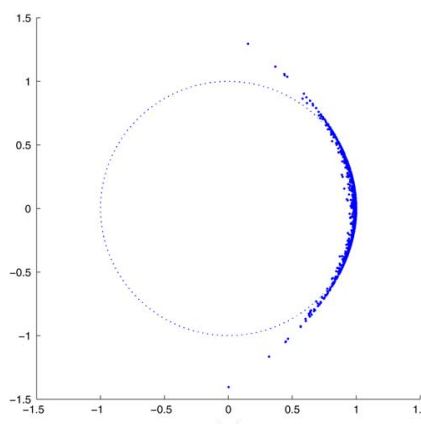

(a)

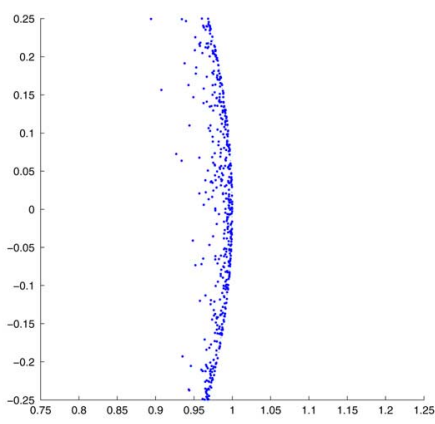

(b)
Fig. 8. Effect of the pseudo-observation method on the unnormalized distribution. The unit circle is drawn as a dashed line and the Monte Carlo samples as points. (a) Overall view. (b) Detailed view.

This theory has been demonstrated by the example in Section III. The original Monte Carlo sample was drawn so that the Type I constraint was satisfied, but the Type II constraint was not satisfied.

The properties of the pseudo-observation algorithm can be assessed in terms of Type I and Type II constraints.

Theorem 2: The pseudo-observation approach applies the Type I constraint only if either a) all samples obey the Type I constraint already or b) if the Type I constraint is linear.

Proof: Consider the pseudo-observation update (5). Applying it to each sample in the distribution gives

$$
\mathbf{x}(k)=\mathbf{x}(k-1)-\mathbf{W}(k) \mathbf{c}[\mathbf{x}(k-1)] .
$$

In the case where the Type I constraint is already obeyed, $\mathbf{c}[\mathbf{x}(k-1)]=\mathbf{0}$; therefore, no update occurs. If the Type I constraint is not obeyed, the sample is moved by a weighted value of the constraint equation. If the translated sample is to obey the nonlinear constraint, it must now obey the condition

$$
\mathbf{c}[\mathbf{x}(k-1)-\mathbf{W}(k) \mathbf{c}[\mathbf{x}(k-1)]]=\mathbf{0} .
$$

This only obeys the result in general if the constraint is linear. This can be seen by substituting the linear projection function (4) determined earlier.

The first part of this theory has been demonstrated by the results in Section III-A. Each sample in the unconstrained estimate was drawn such that the normalization constraint was obeyed. Therefore, when a higher order update strategy was used, the mean did not change. ${ }^{6}$ The second part of this theory is illustrated in Fig. 8(a). One thousand unnormalized samples were drawn at random, and (12) was applied to each one. As can be seen, the points do not lie on the circle. Rather they lie on a parabola (due to the quadratic term in (10)). Near the mean term $(1,0)$, the transformed points appear to lie close to the unit circle. However, Fig. 8(b) shows a close in view. Although many of the samples lie on the unit circle, many lie within it as well.

By contrast, the projection method always guarantees that the Type I constraint is applied. This follows trivially from the definition of the projection operator in (2). However, because of

${ }^{6}$ The covariance was reduced slightly because the UT only approximates terms up to the fourth order and does not capture them completely. 
Jensen's inequality, the mean of this distribution does not lie upon the unit circle but within it.

\section{NEW APPROACH FOR APPLYING NONLINEAR EQUALITY CONSTRAINTS}

The analysis in the previous section shows that there are significant problems with existing algorithms for applying nonlinear constraints: The projection method enforces the Type I constraint, but this does not guarantee that the Type II constraint will be satisfied. The pseudo-observation method does not guarantee that either type of constraint will be satisfied.

We propose that the nonlinear equality constraints should be applied in a two-step process of applying the Type I constraint followed by the Type II constraint.

\section{A. Applying the Type I Constraint}

Suppose the unconstrained estimate has mean $\hat{\mathbf{x}}^{*}(k \mid k)$ and covariance $\mathbf{P}^{*}(k \mid k)$. The first step is to apply the Type I constraint. This constrains the state distribution and, thus, causes the covariance to decrease. As the analysis in the previous section has shown, the pseudo-observation method provides a poor approximation to this constraint. Therefore, the projection method should be applied. Let $\mathbf{x}^{\dagger}(k)$ be the Type I constrained estimate. Therefore

$$
\mathbf{x}^{\dagger}(k)=\mathbf{p}\left[\mathbf{x}^{*}(k)\right]
$$

The mean of the Type I constrained estimate $\hat{\mathbf{X}}^{\dagger}(k \mid k)$ is calculated from

$$
\hat{\mathbf{x}}^{\dagger}(k \mid k)=\mathrm{E}\left[\mathbf{p}\left[\mathbf{x}^{*}(k)\right]\right] .
$$

The covariance $\mathbf{P}^{\dagger}(k \mid k)$ is calculated in a similar manner.

It is important to note that the projection operator can contain multiple degrees of freedom, such as D in (4). The degree of freedom must be chosen to minimize some suitable measure of uncertainty, such as the trace or determinant of $\mathbf{P}^{\dagger}(k \mid k)$.

\section{B. Applying the Type II Constraint}

According to Theorem $1, \hat{\mathbf{x}}^{\dagger}(k \mid k)$ will not, in general, obey the Type II constraint. Therefore, we apply the projection operator to the Type I constrained estimate to generate a new constrained mean

$$
\hat{\mathbf{x}}(k \mid k)=\mathbf{p}\left[\hat{\mathbf{x}}^{\dagger}(k \mid k)\right] .
$$

However, $\hat{\mathbf{x}}^{\dagger}(k \mid k)$ is the constrained estimate with the minimum mean squared error. By moving the estimate to a different value, the filter ceases to propagate the conditional mean, and the covariance must increase so that

$$
\begin{aligned}
\mathbf{P}(k \mid k)=\mathbf{P}^{\dagger}(k \mid k)+(\hat{\mathbf{x}}(k \mid k) & \left.-\hat{\mathbf{x}}^{\dagger}(k \mid k)\right) \\
& \times\left(\hat{\mathbf{x}}(k \mid k)-\hat{\mathbf{x}}^{\dagger}(k \mid k)\right)^{T} .
\end{aligned}
$$

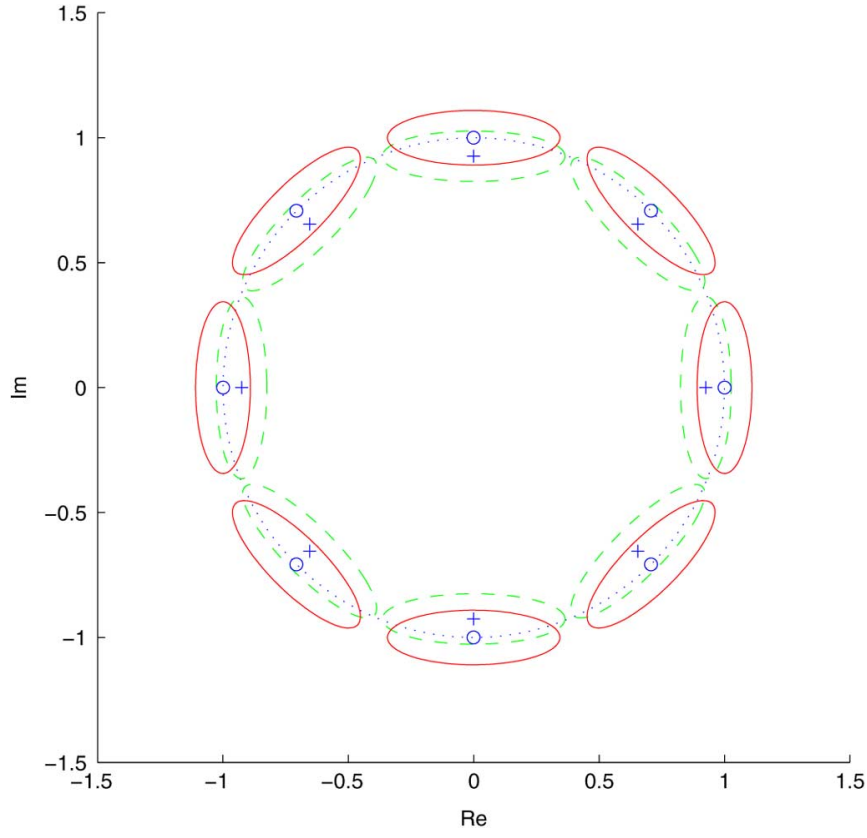

Fig. 9. The $1 \sigma$ contours for estimates constrained using the two-step algorithm. The estimates are shown as solid ellipses with centers at 0 . The unconstrained estimates are the dashed ellipses with centers at + , and the unit circle is the dotted line.

Again, $\mathbf{p}[\cdot]$ may contain free parameters, and these need to be chosen so that the uncertainty in $\mathbf{P}(k \mid k)$ is minimized.

The effect of this two-step process is shown in Fig. 9 using the projection function (11), and the cost metric was to minimize $\left\|\hat{\mathbf{x}}(k \mid k)-\hat{\mathbf{x}}^{\dagger}(k \mid k)\right\|$.

As the figure shows, the mean of the estimate obeys the normalization constraint. The covariances are non-singular and are qualitatively similar in shape to the unconstrained estimates. Fig. 10 shows the effect of the two-step algorithm on 1000 Monte Carlo samples: The Type I constraint projects the points to lie on a circular arc, and the Type II constraint offsets the points such that the mean lies on the unit circle.

We now illustrate this algorithm on a platform tracking example.

\section{Platform Tracking EXAmple}

We consider a vision-based head tracking system. The system is composed of a head-mounted camera that moves through an environment that is populated by a set of landmarks in known locations. The position, orientation, and velocity of the camera is to be estimated based on its observations of those landmarks [23]. Because the camera is head mounted, it can undergo significant linear and angular accelerations.

Orientation is part of the $\mathrm{SO}(3)$ group and can be represented by three parameters using a number of different representations, such as Euler angles [24] and the exponential map [25]. However, all minimal parameterizations possess singularities that can lead to filter instability. The unit quaternion is the lowest dimensional nonsingular attitude representation [13] and has been widely used in augmented reality applications [23], [26]. However, it introduces the nonlinear equality constraint that 


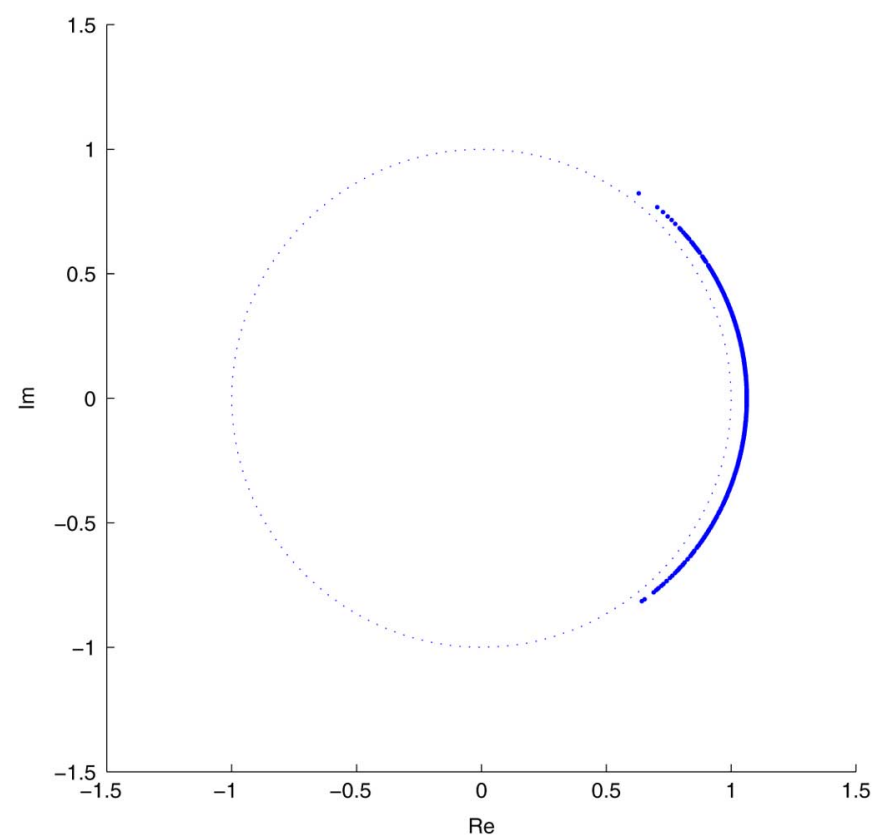

Fig. 10. Effect of the two-step method on the unnormalized distribution. The unit circle is drawn with the dashed line, and the Monte Carlo samples are drawn as points.

the norm of the quaternion states must be one. Hybrid parameterizations, such as the multiplicative extended Kalman filter [13], have been developed that store errors in a three-component vector and global orientation as a unit quaternion. Periodically, the error vector is reset, and its effects are multiplied into the global quaternion. Although these methods automatically guarantee that the unit quaternion is preserved, they do so by reparameterizing the problem and, as explained earlier, do not fall within the scope of this paper.

We use the quaternion representation from Davison [23], and the state space is

$$
\mathbf{x}(k)=\left[\begin{array}{c}
\mathbf{r}(k) \\
\mathbf{q}(k) \\
\mathbf{v}(k) \\
\omega(k)
\end{array}\right]
$$

where $\mathbf{r}(k)=[x y z]^{T}$ is the position, $\mathbf{q}(k)=\left[q_{x} q_{y} q_{z} q_{w}\right]^{T}$ is the orientation (represented as a unit quaternion [27]), $\mathbf{v}(k)=$ $\left[v_{x} v_{y} v_{z}\right]^{T}$ is the velocity, and $\boldsymbol{\omega}(k)=\left[\omega_{x} \omega_{y} \omega_{z}\right]^{T}$ is the angular velocity.

The discrete-time process model is

$$
\mathbf{x}(k+1)=\left[\begin{array}{c}
\mathbf{r}(k)+\Delta t \mathbf{v}(k)+\frac{\Delta t^{2}}{2} \mathbf{a}(k) \\
\mathbf{D}(k) \mathbf{q}(k) \\
\mathbf{v}(k)+\Delta t \mathbf{a}(k) \\
\omega(k)+\Delta t \boldsymbol{\alpha}(k)
\end{array}\right]
$$

where $\mathbf{a}(k)$ is the unknown acceleration, and $\boldsymbol{\alpha}(k)$ is the unknown angular acceleration that acts on the camera at each time step. $\mathbf{D}(k)$ is the skew symmetric matrix given by [10]

$$
\mathbf{D}(k)=\mathbf{I} \cos d+\left[\begin{array}{cccc}
0 & c & -b & a \\
-c & 0 & a & b \\
b & -a & 0 & c \\
-a & -b & -c & 0
\end{array}\right] \frac{\sin d}{d}
$$

where

$$
\begin{aligned}
& a=\frac{\Delta t}{2} \omega_{x}(k)+\frac{\Delta t^{2}}{4} \alpha_{x}(k) \\
& b=\frac{\Delta t}{2} \omega_{y}(k)+\frac{\Delta t^{2}}{4} \alpha_{y}(k) \\
& c=\frac{\Delta t}{2} \omega_{z}(k)+\frac{\Delta t^{2}}{4} \alpha_{z}(k) \\
& d=\sqrt{a^{2}+b^{2}+c^{2}} .
\end{aligned}
$$

Let $\mathbf{R}(k)$ be the rotation matrix encoded by $\mathbf{q}(k)$ and $\mathbf{K}$ be the intrinsic matrix. Let $\mathbf{y}_{i}$ be the location of the $i$ th beacon in 3 -D. The camera observes the $(x, y)$ pixel position of $\mathbf{y}_{i}$ on the imaging plane. This is given by

$$
\mathbf{z}(k)=\left[\begin{array}{c}
\frac{h_{x}}{h_{z}} \\
\frac{h_{y}}{h_{z}}
\end{array}\right]+\mathbf{w}(k)
$$

where

$$
\left[\begin{array}{l}
h_{x} \\
h_{y} \\
h_{z}
\end{array}\right]=\frac{1}{2} \mathbf{K R}(k)\left(\mathbf{y}_{i}-\mathbf{r}(k)\right)
$$

and $\mathbf{w}(k)$ is the additive observation noise.

The camera is a UniBrain Fire-I camera with an $85^{\circ}$ field of view lens and a frame rate of 30 frames/s. $\mathbf{w}(k)$ is a diagonal whose non-zero elements have a standard deviation of 0.2 pixels. Assuming that lens distortion correction has already been applied

$$
\mathbf{K}=\left[\begin{array}{ccc}
883.31 & 1.27586 & 366.132 \\
0 & 883.441 & 211.048 \\
0 & 0 & 1
\end{array}\right]
$$

Because the camera is handheld, it can experience extremely rapid and unmodelled motions. Davison addresses these by using very large values for the process noise standard deviations: $2 \mathrm{~ms}^{-2}$ for linear acceleration and $4 \mathrm{rads}^{-2}$ for angular acceleration. The initial covariance is diagonal. The standard deviations on the positions are $0.1 \mathrm{~m}$, the standard deviations on each quaternion value is $10^{-2}$, the linear velocity $2 \mathrm{~ms}^{-1}$, and angular velocity $3.16 \mathrm{rads}^{-1}$.

The construction of $\mathbf{R}(k)$ assumes that the quaternion is normalized. If the quaternion is not normalized, $\mathbf{R}(k)$ does not correspond to a rotation matrix and, in general, may not have real eigenvalues [13]. Therefore, normalization is extremely important. The constraint and projection functions are

$$
\begin{aligned}
\mathbf{c}[\mathbf{x}(k), k] & =\|\mathbf{q}(k)\|-1 \\
\mathbf{p}[\mathbf{x}(k)] & =\mathbf{q}(k) /\|\mathbf{q}(k)\| .
\end{aligned}
$$

To test the performance of the filters, 2000 Monte Carlo runs were performed, and the average normalized state error was calculated. The normalized state error is defined as

$$
q(k)=(\mathbf{x}(k)-\hat{\mathbf{x}}(k \mid k))^{T} \mathbf{P}^{-1}(k \mid k)(\mathbf{x}(k)-\hat{\mathbf{x}}(k \mid k)) .
$$

Using the normalized state error provides a more precise measure of consistency than looking at state estimates and covariances individually because this metric takes into account the cross correlations between the states as well. The mean value 
TABLE I

Mean Normalized State Errors for all Filters and the Decomposition for Individual Subsets of States. The First Line Gives the Desired

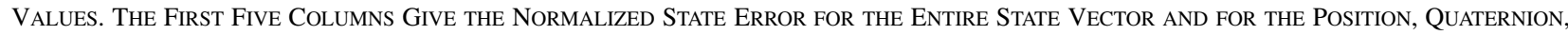
Velocity, and Angular Velocity States. These Were Calculated When the MaXimum AVerage Normalized State ERROR IS 20. The Final Column Lists the Normalized State ERror When the ANGle State Error Threshold was Raised to 100

\begin{tabular}{|c|c|c|c|c|c||c|}
\hline Algorithm & $\mathrm{E}[q(k)]$ & $\mathrm{E}\left[q_{r}(k)\right]$ & $\mathrm{E}\left[q_{q}(k)\right]$ & $\mathrm{E}\left[q_{v}(k)\right]$ & $\mathrm{E}\left[q_{\omega}(k)\right]$ & $\mathrm{E}[q(k)]$ \\
\hline- & 13 & 3 & 4 & 3 & 3 & 13 \\
\hline displOffNoP & $3.2 \times 10^{8}$ & $1.4 \times 10^{4}$ & $4.4 \times 10^{6}$ & $5.6 \times 10^{2}$ & $6.5 \times 10^{4}$ & $3.5 \times 10^{8}$ \\
displOff & 18 & 3.1 & 3.7 & 2.8 & 2.9 & 24 \\
pseudoLinOff & $2.7 \times 10^{14}$ & $2.6 \times 10^{4}$ & $1.9 \times 10^{11}$ & $4.8 \times 10^{2}$ & $1 \times 10^{5}$ & $3.3 \times 10^{14}$ \\
pseudoUnsOff & $3.5 \times 10^{2}$ & 13 & $3.3 \times 10^{2}$ & 3.6 & 3.1 & $1.4 \times 10^{4}$ \\
SCKF & 29 & 4.2 & 6.9 & 2.9 & 3.1 & 54 \\
projLin & $1.1 \times 10^{14}$ & $1.2 \times 10^{5}$ & $9 \times 10^{10}$ & $6.3 \times 10^{3}$ & $1.9 \times 10^{5}$ & $1.3 \times 10^{14}$ \\
projUnsOff & 13 & 2.9 & 3.7 & 2.8 & 2.8 & 15 \\
\hline
\end{tabular}

of $q(k)$ should be the same as the dimensions of the state. In this case, the value is 13 .

Seven filters were implemented where the only difference between them was the algorithm used to enact the normalization step. The algorithms were chosen to compare those found in the literature with our two-step method and to explore the effects of linearization and higher order transformations.

- displOffNoP: This is the simplest method. The Type II constraint is satisfied simply by displacing the mean to esnure that the Type II constraint only is applied. The covariance is unchanged. Therefore

$$
\begin{aligned}
\hat{\mathbf{x}}(k \mid k) & =\mathbf{p}\left[\hat{\mathbf{x}}^{*}(k \mid k)\right] \\
\mathbf{P}(k \mid k) & =\mathbf{P}^{*}(k \mid k) .
\end{aligned}
$$

This approach was used by Davison [23].

- displOff: Like above, the Type II constraint is satisfied by displacing the mean. However, the covariance is updated to reflect the fact that the mean has been displaced

$$
\begin{aligned}
\hat{\mathbf{x}}(k \mid k)= & \mathbf{p}\left[\hat{\mathbf{x}}^{*}(k \mid k)\right] \\
\mathbf{P}(k \mid k)= & \mathbf{P}^{*}(k \mid k)+\left(\hat{\mathbf{x}}(k \mid k)-\hat{\mathbf{x}}^{*}(k \mid k)\right) \\
& \times\left(\hat{\mathbf{x}}(k \mid k)-\hat{\mathbf{x}}^{*}(k \mid k)\right)^{T} .
\end{aligned}
$$

This illustrates the effect of the second step in the two-step process.

- pseudoLinOff: Apply (5) and (6). Because the estimate does not obey the Type II constraint, the displOff algorithm was used to reposition the estimate. We did not use the IEKF approach from Section III-A because the filter always diverged.

- pseudoUnsOff: Apply (5) and (6) but use the unscented transformation. Again, because the estimate does not obey the Type II constraint, the displacement offset algorithm was used to reposition the estimate.

- SCKF: Apply the smoothly constrained Kalman filter [9] for tight constraints using $s_{k \text {,threshold }}^{c}=100$.

- projLin: Apply linearized forms of (7) and (8).

- projUnsOff: This is the two-step algorithm: The projection method is applied using the UT, and the estimate is displaced so that the Type II constraint is satisfied.

Two important limitation were encountered. First, if the angular error becomes large, the filters would diverge, irrespective of the normalization algorithm used. We believe this is a fundamental limitation of using the Kalman filter with a linear update rule and a simple state representation with a single mean and covariance. We found that a simple method for indicating if the angular bounds were exceeded was to test the normalized state error of all filters. If all of them exceeded a minimum value, it was assumed that the angular error constraints were exceeded and the run was excluded from the results. Two thresholds-20 and 100-were used, and the results are discussed below. Second, $q(k)$ exhibited numerical instabilities for the pseudoLinOff and projLin algorithms. These algorithms produced (nearly) singular $\mathbf{P}(k \mid k)$ matrices with extremely large condition numbers. An example of the problem was discussed in Section III-A: The covariance matrix is singular in a direction in which a non-zero error must occur. To generate finite results for comparison purposes, we used a modified form the normalized state error for these filters of the form

$$
\begin{aligned}
q(k)= & (\mathbf{x}(k)-\hat{\mathbf{x}}(k \mid k))^{T}(\mathbf{P}(k \mid k)+\delta \mathbf{N})^{-1} \\
& \times(\mathbf{x}(k)-\hat{\mathbf{x}}(k \mid k))
\end{aligned}
$$

where $\delta=10^{-7}$ is a small constant, and $\mathbf{N}$ is a matrix with ones on the diagonals for states 4-7 (corresponding to the quaternions) and zeros elsewhere.

The performance of the filters was calculated over 2000 Monte Carlo runs, and the means of the normalised state errors, calculated across all runs, are presented in Table I. The two linearized solutions (pseudoLinOff and projLin) perform extremely poorly. The pseudoUnsOff filter performed somewhat better, but its results were still very poor. This illustrates the inadequacies of the the pseudo-observation equations, even when higher order prediction methods are used. The SCKF fares moderately well, but at no point is the quaternion estimate truly normalized-its lower and upper bound lies between 0.98 and $1-10^{-6}$. The two best behaving algorithms were displOff (the second step in the two-step algorithm) and the two-step algorithm itself. The two-step algorithm is the only algorithm that is consistent. To perform a more finegrained analysis of the performance of the filters, we calculated the normalized state errors for the position, quaternion, linear, and angular velocity subvectors only. The results, also shown in Table I, indicate that the effects of the constraint algorithms are most strongly felt by the quaternion estimates themselves. These are strongly tied to the estimates of position and angular velocity. Linear velocity is affected less by the normalization rule.

Because the platform starts from rest and is buffeted by random noise, the variance in the angular and linear velocities 


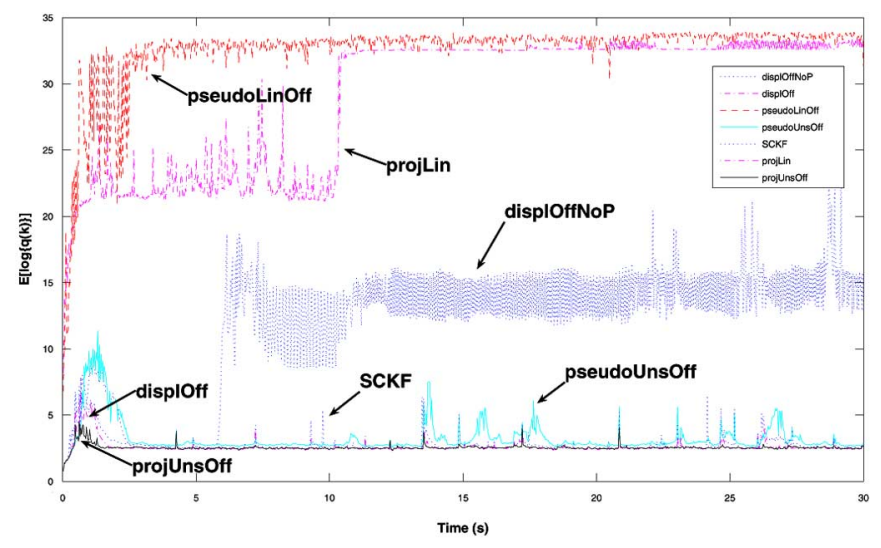

Fig. 11. Natural logarithm of the normalized state error for the different filters. pseudoLinOff and projLin have the largest means, with values about 30. dispOffNoP lies in the middle with a mean of about 15. dispOff, pseudoUnsOff, SCKF, and projUnsOff all have means of around 2.5.

increase over time. To study if these have an effect on the filters, Fig. 11 plots the mean normalized trajectories over the duration of the run. The results show that for most filters, the mean squared error profiles remain about the same through the entire run. However, both the projLin and SCKF filters show step changes: the former at about 10s and the latter at about 6s. We believe that these are caused by repeated divergence of the filters at these timesteps because, at large linear and angular velocities, the effects of errors become more significant.

As explained above, we used a threshold to determine if a run was not valid. The last column of the table shows the results of 2000 Monte Carlo runs when the threshold was increased from 20 to 100 , allowing runs with larger angular errors through. In this situation, the normalized errors in all filters increase, and all filters are inconsistent. However, the projUnsOff filter is less affected than the other filters, indicating that it is more robust than the other algorithms in the presence of large angular errors.

\section{CONCLUSION}

This paper has considered the problem of applying a Kalman Filter-type estimator to a system with nonlinear equality constraints between its state space variables. We have focused on two methods - the pseudo-observation and projection methods-for incorporating these constraints, and we have proposed two different definitions of constraints. Type I constraints define the shape of the entire distribution; Type II constraints only limit the conditional mean propagated in the filter.

We have argued that the pseudo-observation method has little theoretical rigor and enforces neither the Type I nor the Type II constraints. The projection method ensures that the Type I constraint is satisfied, but the Type II constraint is not. To overcome these difficulties and to have a consistent estimate that obeys the Type II constraint, we have proposed a two-step process. In the first step, the Type I constraint is applied using the projection method across the entire distribution. This causes the covariance to reduce. The second step applies the projection method again, this time to the conditional mean, to ensure that the Type II constraint is satisfied. This causes the covariance to increase. We have also demonstrated the performance of the algorithm in a platform tracking example, which is characterized by strong linear and angular accelerations and is thus prone to larger uncertainties. The two-step method was shown to be the only algorithm that was consistent with small angular errors, and when large angular errors are permitted, its performance degrades less severely.

There are a number of avenues for future work. First, the operation of the projection function should be studied in more detail. The current algorithm only moves the states that are directly affected by the constraint. However, all the states should be potentially affected. A similar behavior is observed when inequality constraints are applied [28]. The effect of using the second-order projection operator described in [12] could also be investigated. Second, the effects of different cost functions on the performance of filters should be examined. Third, as we explained in the introduction, there is a third way to circumvent nonlinear equality constraints, which is to reparameterize the system so that they are not needed. A useful area of research would be to compare the performance of different tracking algorithms to see if an over-constrained representation actually provides benefits or not. Finally, the example itself raises the question as to what is the best representation of orientation in head tracking problems. Although many parameterizations of orientation have been proposed, few have been directly compared against one another to ascertain their impact on estimator performance.

\section{ACKNOWLEDGMENT}

The authors would like to thank the reviewers for their many highly useful comments and, in particular, for drawing their attention to the MEKF algorithm [13] and for suggesting some of the proposed work in the conclusions section.

\section{REFERENCES}

[1] E. L. Haseltine and J. B. Rawlings, A critical evaluation of extended Kalman filtering and moving horizon estimation Texas-Wisconsin Modeling and Control Consortium (TWMCC), , Tech. Rep. 2002-03, Mar. 12, 2003

[2] I. Y. Bar-Itzhack and J. Reiner, "Recursive attitude determination from vector observations: DCM identification," J. Guidance, Control Dyn., vol. 7, no. 1, pp. 51-56, -Feb. 1984.

[3] M. Tahk and J. L. Speyer, "Target tracking problems subject to kinematic constraints," IEEE Trans. Autom. Control, vol. 35, no. 3, pp. 324-326, Mar. 1990.

[4] A. T. Alouani and W. D. Blair, "Use of a kinematic constraint in tracking constant speed, maneuvering targets," IEEE Trans. Autom. Control, vol. 38, no. 7, pp. 1107-1111, Jul. 1993.

[5] T. Chia, P. Chow, and H. J. Chizeck, "Recursive parameter identification of constrained systems: An application to electrically stimulated muscle," IEEE Trans. Biomed. Eng., vol. 38, no. 5, pp. 429-442, May 1991.

[6] H. E. Doran, "Constraining Kalman filter and smoothing estimates to satisfy time-varying restrictions," Rev. Econom. Statist., vol. 74, no. 3, pp. 568-572, 1992.

[7] W. Wen and H. F. Durrant-Whyte, "Model-based multi-sensor data fusion," in Proc. IEEE Int. Conf. Robotics Automation. : IEEE Press, May 1992, pp. 1720-1726.

[8] D. Simon and T. Chia, "Kalman filtering with state equality constraints," IEEE Trans. Aerosp.Electron. Syst., vol. 39, no. 1, pp. 128-136, Jan. 2002.

[9] J. D. Geeter, H. V. Brussel, and J. De Schutter, "A smoothly constrained kalman filter," IEEE Trans. Pattern Anal. Machine Intell., vol. 19, no. 10, pp. 1171-1177, Oct. 1997.

[10] R. Azuma, "Predictive Tracking for Augmented Reality," Ph.D. dissertation, Univ. North Carolina, Chapel Hill, 1995.

[11] N. L. Nihan and G. A. Davis, "Recursive estimation of origin-destination matrices from input/output counts," Transport. Res. B, vol. 21B, no. 2, pp. 149-163, 1987. 
[12] C. Yung and E. Blasch, "Kalman filtering with nonlinear state contraints," in Proc. FUSION'06: 9th Int. Conf. Inform. Fusion. Florence, Italy: ISIF, Jul. 10-14, 2006.

[13] L. Markley, "Attitude error representations for Kalman filtering," $J$. Guidance, Control Dyn., vol. 26, no. 2, pp. 311-317, 2003.

[14] Y. Bar-Shalom and T. E. Fortmann, Tracking and Data Association. New York: Academic, 1988.

[15] T. Chia, "Parameter Identification and State Estimation in Constrained Systems," Ph.D. dissertation, Case Western Reserve Univ., Cleveland, $\mathrm{OH}, 1986$.

[16] D. Choukroun, "Novel Methods for Attitude Determination Using Vector Observations," Ph.D. dissertation, Technion-Israel Inst. Technol., Haifa, Israel, May 2003.

[17] H. F. Durrant-Whyte, "Uncertain geometry in robotics," IEEE J. Robotics Automat., vol. 4, no. 1, pp. 23-31, Feb. 1988.

[18] R. L. Wheeden and A. Zygmund, Measure and Integral: An Introduction to Real Analysis. : Marcel Dekker, 1977.

[19] R. L. Bellaire, E. W. Kamen, and S. M. Zabin, "A new nonlinear iterated filter with applications to target tracking," in Proc. AeroSense: 8th Int. Symp. Aerospace/Defense Sensing, Simulation and Controls, Orlando, FL, Apr. 1995, vol. 2561, pp. 240-251.

[20] A. H. Jazwinski, Stochastic Processes and Filtering Theory. San Diego, CA: Academic, 1970.

[21] S. J. Julier, J. K. Uhlmann, and H. F. Durrant-Whyte, "A new approach for the nonlinear transformation of means and covariances in filters and estimators," IEEE Trans. Automat. Control, vol. 45, no. 3, pp. 477-482, Mar. 2000.

[22] J. D. Geeter, Personal Communication Feb. 2004.

[23] A. J. Davison, "Real-time simultaneous localisation and mapping with a single camera," in Proc. Int. Conf. Computer Vision (ICCV), Nice, France, Oct. 13-16, 2003.

[24] E. Foxlin, "Inertial head-tracker sensor fusion by a complementary separate-bias kalman filter," in Proc. IEEE Virtual Reality Annu. Symp., Santa Clara, CA, Mar. 30-Apr. 3 1996, pp. 185-194.

[25] F. S. Grassia, "Practical parameterization of rotations using the exponential map," J. Graphics Tools, vol. 3, no. 3, pp. 29-48, 1998.

[26] R. Azuma and G. Bishop, "Improving static and dynamic registration in an optical see-through HMD," in Proc. SIGGRAPH '94, Jul. 1994, pp. 197-204.

[27] J. B. Kuipers, Quaternions and Rotation Sequences. Princeton, NJ: Princeton Univ. Press, 1999.
[28] N. Shimada, Y. Shirai, Y. Kuno, and J. Miura, "Hand gesture estimation and model refinement using monocularcamera-ambiguity limitation by inequality constraints," in Proc. Third IEEE Int. Conf. Automatic Face Gesture Recognition, Nara, Japan, Apr. 14-16, 1998, pp. 268-273.

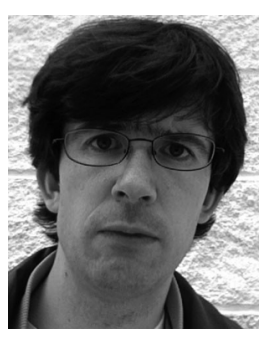

Simon J. Julier (M'93) is a Senior Lecturer at the Vision, Imaging, and Virtual Environments Group, Computer Science Department, University College London (UCL), London, U.K. Before joining UCL, he worked for nine years at the 3D Mixed and Virtual Environments Laboratory, Naval Research Laboratory, Washington, DC, where was PI of the Battlefield Augmented Reality System (BARS): a research effort to develop man-wearable systems for providing situation awareness information. $\mathrm{He}$ served as the Associate Director of the 3DMVEL from 2005 to 2006. His research interests include user interfaces, distributed data fusion, nonlinear estimation, and simultaneous localization and mapping.

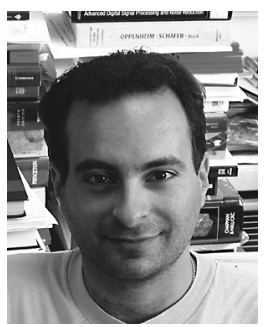

Joseph J. LaViola, Jr. (M'06) received the SC.M degree in computer science in 2000 , the SC.M. degree in applied mathematics in 2001, and the Ph.D. degree in computer science in 2005, all from Brown University, Providence, RI.

$\mathrm{He}$ is an assistant professor with the School of Electrical Engineering and Computer Science, the University of Central Florida, Orlando, as well as an adjunct assistant research professor with the Computer Science Department, Brown University. His primary research interests include pen-based interactive computing, 3D interaction techniques, predictive motion tracking, multimodal interaction in virtual environments, and user interface evaluation. His work has appeared in journals such as Presence and IEEE COMPUTER GRAPHICS AND APPLICATIONS, and he has presented research at conferences including ACM SIGGRAPH, the ACM Symposium on Interactive 3D Graphics, IEEE Virtual Reality, and Eurographics Virtual Environments. He has also co-authored 3D User Interfaces: Theory and Practice, which is the first comprehensive book on 3D user interfaces. 